\title{
Analyse der Sanierungsmaßnahmen an zwei Hydrogeneratoren mit AGS/EGS-Schäden anhand diagnostischer Ozonmessungen
}

\author{
B. Windisch
}

angenommen am 18. Oktober 2021, online publiziert am 2. November 2021

(c) Springer-Verlag GmbH Austria, ein Teil von Springer Nature 2021

Ozonmessungen und visuelle Kontrollen zeigten an den Statorwicklungen zweier Maschinen der VERBUND Hydro Power GmbH Schäden im Bereich des Nutaustritts am AGS/EGS-Übergang auf. Die daraufhin durchgeführten Sanierungsmaßnahmen wurden an beiden Maschinen anhand abschließender Ozonmessungen bewertet und zeigten durchaus konträre Ergebnisse hinsichtlich des Sanierungserfolges.

An zwei Synchrongeneratoren unterschiedlicher Bauform (Vertikal - bzw. Rohrmaschine) der VERBUND-Flotte förderten Diagnosemessungen und visuelle Inspektionen ähnliche Schadensbilder im Bereich des Nutaustritts im Übergangsbereich AGS/EGS zutage. Beide Maschinen zeigten fortgeschrittene Erosionen und waren hinsichtlich ihrer Vorgeschichte bereits dafür bekannt, Probleme im genannten Bereich aufzuweisen. Die Sanierungsmaßnahmen an beiden Maschinen wurden vom selben fachkundigen Unternehmen durchgeführt und waren an beiden Wicklungen annähernd ident.

Die betroffenen Stellen wurden gereinigt und loses Material entfernt, anschließend erfolgten jeweils ein erster und zweiter Anstrich des AGS wie auch des EGS. Danach wurden der AGS durch EWBSilikonmasse an das Blechpaket angebunden und die Sanierungsstellen lackiert.

Bei den abschließenden Ozonmessungen zur Überprüfung des Sanierungserfolgs wurden lediglich an einer Maschine signifikant niedrigere Werte gemessen, die zweite Maschine zeigte ähnlich hohe
Werte wie vor der Sanierung. Die Analyse der gemessenen Ozonwerte legt den Schluss nahe, dass bei der Maschine mit unverändert hohen Messwerten auch im nicht sanierten Nutbereich von massiven AGS-Schäden ausgegangen werden muss und die Sanierung nur einen minimalen Bereich der geschädigten Wicklungsoberfläche erfassen konnte.

Die zweite Maschine hingegen zeigte nach der Sanierung zufriedenstellende Ozonmesswerte. Hier kann davon ausgegangen werden, dass die Sanierung erfolgreich war und alle groben Schadstellen ausgebessert wurden. Interessant ist hierbei auch ein Vergleich mit der Schwesternmaschine des erfolgreich sanierten Generators. Diese zeigte bei einer kürzlich durchgeführten Ozonanalyse ebenfalls alarmierende Werte, und es kann davon ausgegangen werden, dass sich bei einer visuellen Inspektion ein ähnliches Schadensbild wie an der Schwesternmaschine (vor der Sanierung) zeigen wird.

Der Vergleich der beiden sanierten Generatoren hinsichtlich des Reparaturerfolges zeigt, dass eine begleitende diagnostische Bewertung bei Sanierungsmaßnahmen sehr sinnvoll ist und einen wertvollen Beitrag zum Asset-Management leisten kann.

Hinweis des Verlags Der Verlag bleibt in Hinblick auf geografische Zuordnungen und Gebietsbezeichnungen in veröffentlichten Karten und Institutsadressen neutral
Kurzfassung eines Vortrags bei der OVE-Energietechnik-Tagung, 58. Fachtagung de Energietechnik-Branche, die am 6. und 7. Oktober 2021 in Linz stattfand.

Windisch, Bernhard, VERBUND Hydro Power GmbH, Europaplatz 2, 1150 Wien, Österreich (E-Mail: bernhard.windisch@verbund.com) 